\title{
Study on Adaptive Slid Mode Controller for Improving Handling Stability of Motorized Electric Vehicles
}

\author{
LiQiang Jin and Yue Liu \\ State Key Laboratory of Automotive Dynamic Simulation, Jilin University, Changchun 130022, China \\ Correspondence should be addressed to Yue Liu; liuyueresearch@163.com
}

Received 21 September 2014; Revised 4 November 2014; Accepted 4 November 2014; Published 26 November 2014

Academic Editor: Hui Zhang

Copyright (C) 2014 L. Jin and Y. Liu. This is an open access article distributed under the Creative Commons Attribution License, which permits unrestricted use, distribution, and reproduction in any medium, provided the original work is properly cited.

\begin{abstract}
An adaptive slid mode controller was established for improving the handling stability of motorized electric vehicle (MEV). First and foremost, the structure and advantages of electric vehicle driven by in-wheel motors will be provided. Then, an ideal cornering model of vehicles will be brought and analyzed, after which a method to estimate side-slip angle was also proposed and three typical sensors were used in the theory. Besides, an idea for the recognition of road adhesion coefficient was derived based on MEV platform, which will be helpful for better control performances. Finally, the scheme of control method was given and some typical tests for observing handling properties were implemented based on Simulink and Carsim software. With the outcomes from the experiments, which vividly showed the merits of the controller, one can come to a conclusion that MEV that equips with the adaptive slid mode controller always enjoys better handling performances than the one without control. Furthermore, the controller researched is friendly to the real-time working conditions, which will hold practical values in the future.
\end{abstract}

\section{Introduction}

Scarcity of fuel has brought in great concerns to human today. Gasoline, which has been the major power source of classical vehicles for decades, got its price soaring up year by year. This phenomenon results in an unstoppable trend of conventional cars being replaced by vehicles powered not by gasoline but by another new energy, such as Electric Vehicles. However, the cost by devices of Electric Vehicles is always considerable. Electric Vehicles driven by in-wheel motors present to us a practical way of designing an Electric Vehicle with its cost reduced $[1,2]$. In addition, this system is considered to be the universal driving platform of vehicles including EV, HEV, and FCEV. It is the evolution trend of the new generation electric vehicle driving systems. Many novel models have been promoted into the market by famous automotive manufacturers such as Mitsubishi, Honda, Ford, GM, and Volvo [3, 4].

The performance of in-wheel motor Electric Vehicle is likely to perform better compared with that of classical vehicles once a good control system is invented [5-7]. Energy from kinetic of electric vehicle can be recovered by motors during braking process, which is a remarkable feature of great use and of great value in the future [8]. The torque of the motor can be unleashed immediately and accurately. The properties of torque with these features were demonstrated by some researchers [9]. Steering assistance by differential torque that generated by the left and right wheel motors in MEV has also been studied [10], in which the hand-wheel torque was lower down without additional complex devices (such as power assisted steering systems).

Owing to the attractive performance of active safety technologies such as Antilock Braking System (ABS), Traction Control System (TCS), and Electronic Stability Control (ESC), the manipulating stability and safety of vehicles were increased enormously [11-13] and, thus, some developed countries in Europe and North America have already set the requirement of electric stability control system into the rule as the standard equipment in vehicles [14]. Considerable efforts have been made to improve the handling stability of classical vehicles and MEV. Zhang et al. proposed a robust gainscheduling energy-to-peak control strategy with both varied longitudinal velocity and equivalent cornering stiffness taken into consideration. Owing to that idea, handling stability and safety of vehicles were intensified [15]. Shuai et al. have been investigating the combination of AFS and DYC in 4WID-EVs 


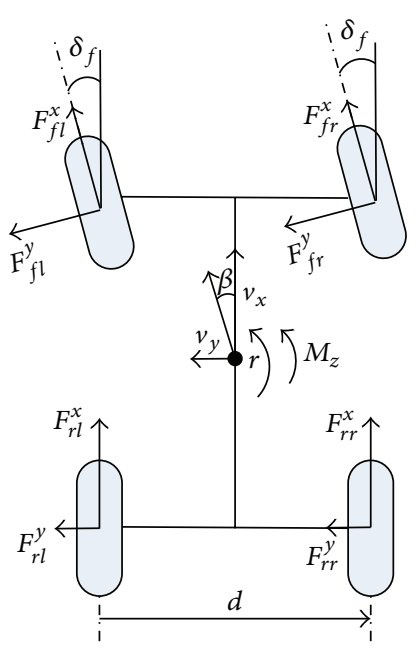

(a)

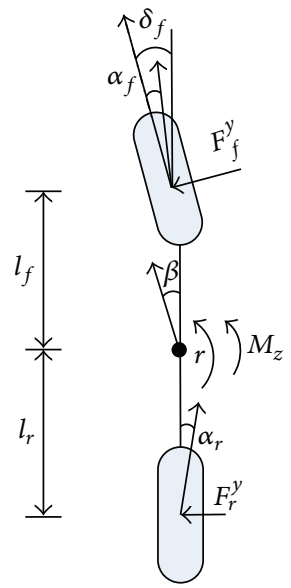

(b)

FIGURE 1: Vehicle dynamic model when cornering.

owing to the flexibility and possibility of fully utilizing the adhesion of each tire [16-18]; the theory was demonstrated to be friendly in stability control. A novel RWG $\mathrm{H}_{\infty}$ controller is developed to control vehicle lateral motions by Huang et al. [19]; the idea attenuated the adverse effects of the steering system hysteresis on the tracking of yaw rate references and the performances of handling stability do be pretty good. The reason why vehicles perform unstably in certain situations is that properties of tires are nonlinear; Umeno et al. have been studying this phenomenon and proposed a control method for improving vehicle stability based on the side-slip angle [12]. It demonstrates that the side-slip angle will be sharply increased when cornered with the mutation of steering angle. In addition, the value of yaw moment will be closed to zero at the same time, which means that the adhesion condition of tires has already reached its limit. Vehicles are likely to become unstable when side-slip angle is over $12 \mathrm{deg}$ if the road is dry, but if the road is wet, the vehicle will definitely be out of control when the side-slip angle is over $5 \mathrm{deg}$. Forster noted that vehicles including tires and suspensions must be made to fit for human's behaviors. Vehicles must be in control even when it has got severely deviated from normal track $[13,14]$. The control themes, which hold practical values, are known to be divided into two kinds. One considers yaw rate only, such as DSC (Dynamic Stability Control) system of BMW. The other one takes both yaw rate and side-slip angle into consideration, such as the VDC (Vehicle Dynamic Control) system of BOSH and the VSC (Vehicle Stability Control) system of Toyota [20-23].

From the discussion above, it is evident that the major point in stabilization control is to identify both yaw rate and side-slip angle in real-time. In order to design a reasonable controller which can keep vehicle body always stable, those two parameters must be taken into consideration simultaneously. Besides, the sensor that used to monitor yaw rate is very common; however, the sensor which is used to monitor the side-slip angle is always expensive [3].
In this study, an adaptive slid mode controller integrated with both yaw rate and side-slip angle will be provided to enhance the handling stability of MEV. A method for road adhesion factor estimation will also be proposed. Besides, an estimation idea of side-slip angle is established in order to lower the cost of the sensors. Finally, two kinds of typical experiments, the double lane change test and $U$ turn test, were carried out by using Simulink and Carsim software. The merits of the adaptive slid mode controller were verified vividly through comparison of the results. In addition, the algorithm is very simple and easy to be implemented, which means that the controller is fit for real time working conditions and, thus, will have practical value in the future.

\section{Vehicle Model and Deduction of Desired Parameters}

2.1. Ideal Vehicle Model and Analysis. As shown in Figure 1(a), which is a simplified MEV model when When the vehicle is cornering the equations can be given by

$$
\begin{aligned}
& m\left(\dot{v}_{y}+v_{x} \gamma\right) \\
& =\left(F_{f l}^{x} \sin \delta_{f}+F_{f l}^{y} \cos \delta_{f}\right) \\
& \quad+\left(F_{f r}^{x} \sin \delta_{f}+F_{f r}^{y} \cos \delta_{f}\right)+\left(F_{r l}^{y}+F_{r r}^{y}\right), \\
& I_{z} \dot{\gamma} \\
& =l_{f}\left[\left(F_{f l}^{x} \sin \delta_{f}+F_{f l}^{y} \cos \delta_{f}\right)+\left(F_{f r}^{x} \sin \delta_{f}+F_{f r}^{y} \cos \delta_{f}\right)\right] \\
& \quad-l_{r}\left(F_{r l}^{y}+F_{r r}^{y}\right)+M_{z},
\end{aligned}
$$

where $m$ is the mass of MEV; $v_{x}$ and $v_{y}$ are the longitudinal velocity and lateral speed; $F_{i}^{x}$ and $F_{i}^{y}$ stand for the longitudinal forces and lateral forces that are generated by each tire; 


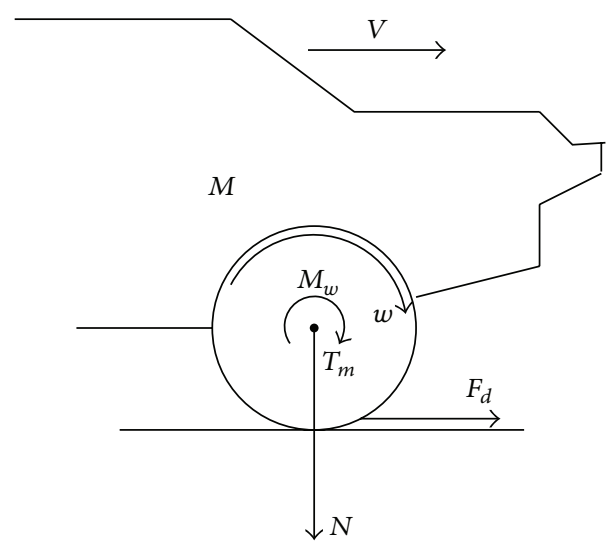

FIGURE 2: Quarter model of vehicle.

$\delta_{f}$ is the wheel angle inputs by the driver; $l_{i}$ is vehicle size as shown in Figure 2; to be mentioned, $M_{z}$ is the coordination torque that is governed by the four driving wheels. $M_{z}$, which is regarded as stability control torque, can be calculated as

$$
M_{z}=\frac{d}{2}\left(F_{r r}^{x}-F_{r l}^{x}\right)+\frac{d}{2}\left(F_{f r}^{x}-F_{f l}^{x}\right) \cos \delta_{f} .
$$

In this study, Provided that the relationship between the front in-wheel motors and the rear in-wheel motors can be described as (4) when generating the yaw control moment

$$
\left(F_{r r}^{x}-F_{r l}^{x}\right)=\varepsilon\left(F_{f r}^{x}-F_{f l}^{x}\right) .
$$

The value of $M_{z}$ is 0 when the vehicle is driving stably; however, the value will be generated when the vehicle becomes unstable. Obviously, by having feasible control of $M_{z}$, one can get a stable working environment when cornering. Conventional vehicles, however, cannot generate this torque without any other auxiliary devices because of the differentials.

2.2. Desired Yaw Rate. To simplify the model, a single track model is introduced like Figure 1(b); the equilibrium equations then can be expressed:

$$
\begin{aligned}
& m v_{x}(\dot{\beta}+\gamma)=F_{f}^{y} \cos \delta_{f}+F_{r}^{y}, \\
& I_{z}=l_{f} F_{f}^{y} \cos \delta_{f}-l_{r} F_{r}^{y}+M_{z} .
\end{aligned}
$$

Assuming vehicle was driving in ideal working conditions, namely, the lateral tire forces are linearly changed, then the tire forces generated on front wheel and rear wheel can be deduced as

$$
\begin{aligned}
& F_{f}^{y}=-2 C_{f}\left(\beta+\frac{l_{f} \gamma}{v_{x}}-\delta_{f}\right), \\
& F_{r}^{y}=-2 C_{r}\left(\beta-\frac{l_{r} \gamma}{v_{x}}\right) .
\end{aligned}
$$

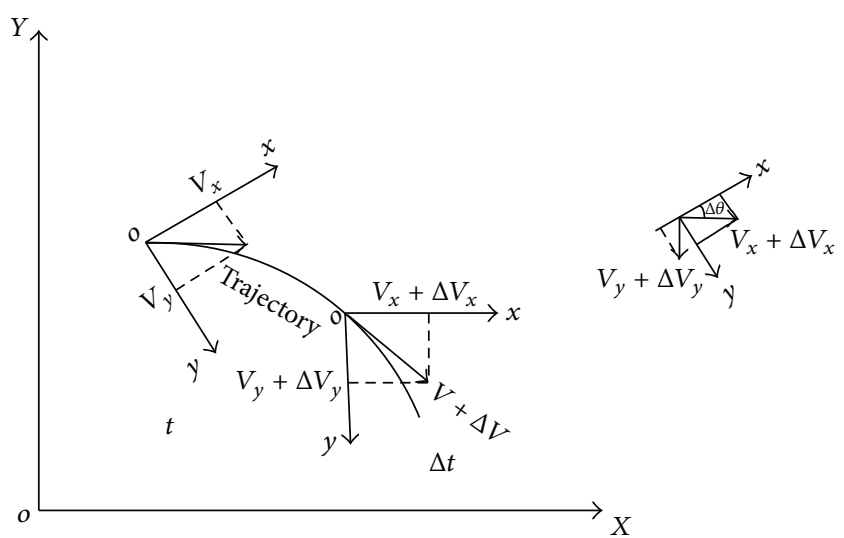

Figure 3: Analysis of vehicle trajectory.

Provided that vehicle was driving in a stable working condition in which the value of the parameters can be seen as

$$
\dot{\beta}=\dot{\gamma}=0,
$$

then the ideal yaw rate can be expressed as

$$
\gamma_{\text {desired }}^{\prime}=\frac{v_{x}}{L+\left(W_{f} / C_{f}-W_{r} / C_{r}\right)\left(v_{x}^{2} / g\right)} \delta_{f},
$$

where $W_{i}$ is the vertical load on each tire; $C_{i}$, which can be seen as a constant value ideally, stands for the lateral tire stiffness.

The desired yaw rate, however, also is limited by the road adhesion factor. When vehicle corners, lateral acceleration under vehicle coordinate system (as shown in Figure 3) can be expressed as

$$
a_{y}=\dot{x} \gamma+\ddot{y} .
$$

In addition, the relationships among the side-slip angle and velocities can be deduced:

$$
\tan \beta=\frac{\dot{y}}{\dot{x}} .
$$

Then lateral acceleration can be expressed as

$$
a_{y}=\dot{x} \gamma+\ddot{x} \tan \beta+\frac{\dot{x} \dot{\beta}}{\sqrt{1+\tan ^{2} \beta}} .
$$

Define $\mu$ as road adhesion coefficient, since the quadratic integral term can be ignored when side-slip angle is very small. Provided that the contribution value of the latter two equations is 0.1 , then we can get

$$
a_{y} \approx 1.1 \dot{x} \gamma \leq \mu g .
$$

So the limitation can be derived as

$$
\gamma_{\lim } \approx 0.9\left|\frac{g \cdot \mu}{V_{x}}\right| .
$$


From all the equations above, the ideal output of yaw rate can be defined as

$$
\gamma_{\text {desired }}=\min \left\{\gamma_{\text {desired }}^{\prime}, \gamma_{\text {lim }}\right\}
$$

2.3. Desired Side-Slip Angle and the Usage of the Parameter. In general, the value of the desired side-slip angle is the smaller, the better. For most research today, the desired value is considered to be $0^{\circ}$. In this paper, both yaw rate and sideslip angle were included. The controller will keep on working since the value of side-slip angle is not zero in a real working condition. In general, vehicle in high adhesion road (assume $\mu=0.9$ ) will become unstable when the value of side-slip angle is over $12^{\circ}$; however, the value is only $5^{\circ}$ in low adhesion road (assume $\mu=0.35$ ).

In this research, it is necessary to define a weighing factor $\varsigma$ to describe the contribution value of the side-slip angle. Logic threshold method was chosen to decide the value of the weighing factor. For instance, in low friction $\operatorname{road}(\mu=0.35)$, the factor is 0 when side-slip angle is smaller than $4^{\circ}$, while the factor is 1 when the value is bigger than $5^{\circ}$; the value between them is linearly changed. Generally, the threshold of different roads can be expressed as a function

$$
\begin{aligned}
& \beta_{\text {start }}=\arctan (0.02 \mu \mathrm{g}), \\
& \beta_{\text {high }}=\arctan (0.025 \mu \mathrm{g}) .
\end{aligned}
$$

Then the value change of weighing factor based on the logic threshold theory is identified by

$$
\begin{gathered}
\varsigma=0 \quad \text { if } \beta<\arctan (0.02 \mu g) \\
\varsigma=\frac{\beta}{\arctan (0.025 \mu \mathrm{g})} \quad \text { if } \arctan (0.025 \mu \mathrm{g}) \\
<\beta<\arctan (0.02 \mu \mathrm{g}) \\
\varsigma=1 \quad \text { if } \beta>\arctan (0.025 \mu \mathrm{g}) .
\end{gathered}
$$

\section{Identification of Road Adhesion Factor of Electric Vehicle with Motorized Wheels}

As we can see the two desired parameters are related to the value of road adhesion factor, so it is necessary to estimate the adhesion coefficient accurately. In order to resolve the differential problem, the driving motor of electric vehicle is controlled by torque mode $[24,25]$. A quarter vehicle model is shown in Figure 2.

The equation for wheel and body dynamics can be expressed by Newton's law as follows:

$$
\begin{aligned}
& I_{w} \frac{d w}{d t}=T_{m}-F_{d} \cdot r, \\
& M \frac{d V}{d t}=F_{d}, \\
& u(s)=\frac{F_{d}}{N},
\end{aligned}
$$

where $T_{m}$ is the torque that transmitted from the motor; $F_{d}$ is the friction force between road and tire; $I_{w}$ is the rotating inertia of wheel; $M$ is part of the vehicle mass that acts on single wheel; $w$ is the rotating velocity of the wheels; $V$ is the body velocity; $N$ is the vertical load generated by $M ; r$ is the tire radius. Then $\mu$ can be derived:

$$
\mu=\frac{1}{N r}\left(T_{m}-I_{w} \frac{d w}{d t}\right) .
$$

$\mu$, which is defined as the adhesion coefficient between tire and road, can be obtained when $T_{m}$ and $w$ are easily and precisely identified. The formula is also suitable for traditional cars. In traditional cars, however, it is very difficult to gain the accurate driving torque of each wheel in real-time as well as estimate the friction characteristics based on (18) owing to the complex transmission system. But for the electric vehicle driven by in-wheel motors, the torque and velocity on the wheel can be easily measured, so the road adhesion can be accurately estimated if the vehicle is driven by in-wheel motors.

\section{Side-Slip Angle Estimation}

For a classical ESC system, some basic sensors such as yaw rate sensor and body acceleration sensors were included. The main parameter like side-slip angle, which was crucial for stability control, seems hard to be obtained. Most estimation methods today are based on Kalman filter [26, 27]; however, the idea was bad for real-time working conditions due to the long sampling time. For ESC system, quick response is needed. So an estimation method will be studied in order to solve the problem and reduce the cost of the sensors. As seen from Figure 3, vehicle was simplified as the center of its mass. Assume that vehicle moves in extremely short time $\Delta t$.

The trajectory was in vehicle coordinate system $y$ - $x$. The velocity in $x$ direction can be expressed as

$$
\left(V_{x}+\Delta V_{x}\right) \cos \Delta \theta-V_{x}-\left(V_{y}+\Delta V_{y}\right) \sin \Delta \theta
$$

$\Delta \theta$ is assumed to be zero in extremely short time. So the equation can be simplified as

$$
\Delta V_{x}-V_{y} \Delta \theta
$$

According to the definition of differential in mathematics, acceleration in $x$ direction is

$$
a_{x}=\frac{d V_{x}}{d t}-V_{y} \frac{d \theta}{d t}=\dot{V}_{x}-V_{y} \gamma .
$$

Similarly, acceleration in $y$ direction can be deduced as

$$
a_{y}=\dot{V}_{y}-V_{x} \gamma
$$

The side-slip angle of automobile can be depicted as

$$
\beta=\arctan \left(\frac{V_{y}}{V_{x}}\right),
$$


where $V_{x}$ and $V_{y}$ were the velocities of vehicle, $\Delta \theta$ was the turning angle in that infinitesimal time. $\beta$ can be identified from (21)-(23) with the value of $a_{x}, a_{y}, \gamma$ that was obtained from the three basic sensors. The idea proposed is friendly to the real-time working conditions, since there is no sampling time needed.

\section{Control Algorithm for Enhancing Handling Stability}

The changing of slid mode controller is purposeful based on the working conditions of the system, Which forces the systems run according to a predetermined "sliding mode" trajectory. Slid mode control holds the merits of fast response, being insensitive to parameter changes and disturbances, having no need to identify system online, and the ability to be very easily implemented [28].

Based on (1)-(4), we choose sliding surface as

$$
S=\gamma-\gamma_{\text {desired }}+\varsigma\left(\beta-\beta_{\text {desired }}\right)
$$

Equation (2) can be rewritten as (25) since the value of $\delta_{f}$ is usually very small:

$$
I_{z} \dot{\gamma}=l_{f}\left(F_{f l}^{y} \cos \delta_{f}+F_{f r}^{y} \cos \delta_{f}\right)-l_{r}\left(F_{r l}^{y}+F_{r r}^{y}\right)+M_{z}
$$

Then based on (3) and (4), the formula can be expressed as

$$
\begin{aligned}
I_{z} \dot{\gamma}= & l_{f}\left(F_{f l}^{y} \cos \delta_{f}+F_{f r}^{y} \cos \delta_{f}\right) \\
& -l_{r}\left(F_{r l}^{y}+F_{r r}^{y}\right)+M_{c}\left(\cos \delta_{f}+\varepsilon\right),
\end{aligned}
$$

where $M_{c}=(d / 2)\left(F_{f r}^{x}-F_{f l}^{x}\right)$ is the control torque that needed to be sent to the torque distributor and then to the motors. The differential version of (24) can be deduced as

$$
\dot{S}=\dot{\gamma}-\gamma_{\text {desired }}+\varsigma\left(\dot{\beta}-\beta_{\text {desired }}\right) \text {. }
$$

Substituting for $\gamma$ in equation (26)

$$
\begin{aligned}
\dot{S}=\left\{\frac { 1 } { I _ { z } } \left[l_{f}\left(F_{f l}^{y} \cos \delta_{f}+F_{f r}^{y} \cos \delta_{f}\right)-l_{r}\left(F_{r l}^{y}+F_{r r}^{y}\right)\right.\right. \\
\left.\quad+M_{c}\left(\cos \delta_{f}+\rho\right)\right] \\
\left.-\gamma_{\text {desired }}+\varsigma\left(\dot{\beta}-\beta_{\text {desired }}^{\cdot}\right)\right\} .
\end{aligned}
$$

Here we choose the exponent law that yields the control

$$
\dot{S}=-\eta S,
$$

where $\dot{S} S<0$ means that the system will be stable based on Lyapunov criteria.
Then the control torque can be deduced as

$$
\begin{aligned}
M_{c}= & \frac{I_{z}}{\left(\cos \delta_{f}+\varepsilon\right)} \\
& \times\left\{\gamma_{\text {desired }}-\eta S-\varsigma\left(\dot{\beta}-\beta_{\text {desired }}^{\cdot}\right)+\frac{1}{I_{z}}\right. \\
& \left.\quad \times\left[l_{r}\left(F_{r l}^{y}+F_{r r}^{y}\right)-l_{f}\left(F_{f l}^{y} \cos \delta_{f}+F_{f r}^{y} \cos \delta_{f}\right)\right]\right\} .
\end{aligned}
$$

Since the load of the tire is not easy to monitor. Here, replace $\left(1 / I_{z}\right)\left[l_{r}\left(F_{r l}^{y}+F_{r r}^{y}\right)-l_{f}\left(F_{f l}^{y} \cos \delta_{f}+F_{f r}^{y} \cos \delta_{f}\right)\right]$ by $-k \int s d t$, which means an integral error feedback term, where $k$ is integral factor. Then we have

$$
\begin{aligned}
M_{c}= & \frac{I_{z}}{\left(\cos \delta_{f}+\varepsilon\right)} \\
& \times\left\{\gamma_{\text {desired }}-\eta S-\varsigma\left(\dot{\beta}-\beta_{\text {desired }}\right)-k \int s d t\right\} .
\end{aligned}
$$

After calculating the value of $M_{c}$ in each step, the torque of the left motor will become smaller than the right one if $M_{c}>0$, so it is the same idea that lowers down the right motor torque when $M_{c}<0$.

Then the adaptive slid mode control strategy in this study can be depicted as in Figure 4. As shown in the diagram, sensors attached on the vehicle will monitor the condition parameters every step time and calculate the desired handling variables; then the slid mode surface function will be obtained based on (31), from which formula of the yaw control moment will be generated.

Then the driving motors will have a feasible response that try to make the car maintain stability. As we can see, the value of weighing factor $\varsigma$ is changed based on the side-slip angle and road adhesion coefficient, which means that the controller can adapt to different kinds of working conditions in real-time.

\section{Typical Simulation Experiments and Results}

For the validation, two typical maneuvers will be considered here. The first maneuver is double-lane change test, whose path is controlled by a prescribed steering input. The second maneuver is $\mathrm{U}$ turn test. The two kinds of experiments, which can describe vehicles' general properties in extreme cases, are widely used in handling stability test, for instance, the obstacle avoidance or changing path when driving on the overpass. Parameters of the test vehicles are shown in Table 1. The maximum power of the motor is $25 \mathrm{~kW}$ and maximum torque is $400 \mathrm{~N} \cdot \mathrm{m}$, as shown in Table 2. The simulation test will be carried out based on Simulink and Carsim software. The controller will be built in Simulink, while MEV model will be generated by Carsim software.

6.1. Double Lane Change Test. The basic test conditions are listed as follows (Figure 5): 


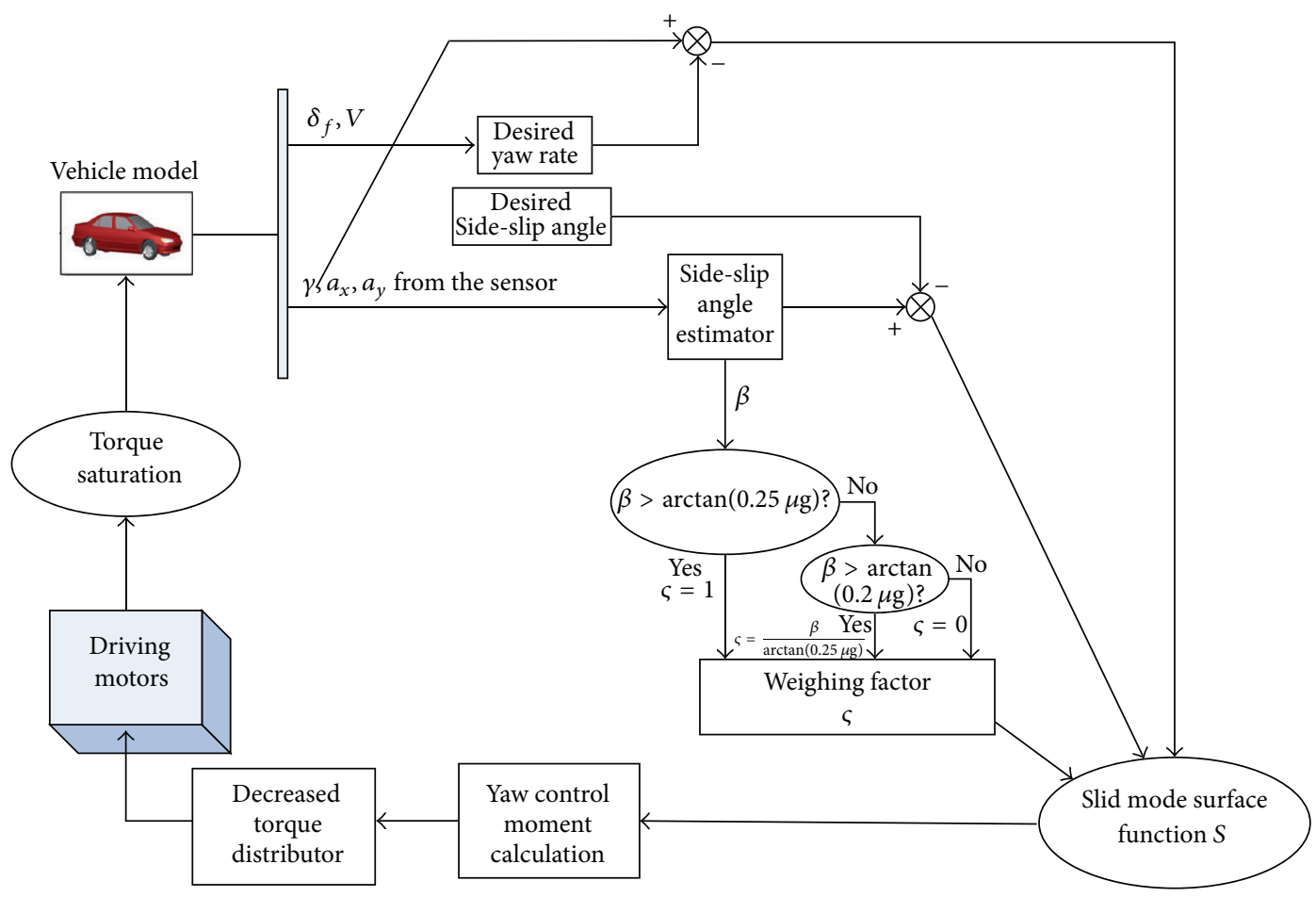

FIGURE 4: Control scheme of adaptive slid mode controller.

TABLE 1: Vehicle specification for simulation.

\begin{tabular}{lclc}
\hline Total mass & $1712 \mathrm{~kg}$ & Wheelbase & $2.95 \mathrm{~m}$ \\
Wheel inertia & $9.11 \mathrm{~kg} \cdot \mathrm{m}^{2}$ & Tread & $1.85 \mathrm{~m}$ \\
Wheel mass & $60 \mathrm{~kg}$ & Wheel radius & $0.385 \mathrm{~m}$ \\
\hline
\end{tabular}

TABLE 2: Main specification for the driving motor.

\begin{tabular}{lclc}
\hline Rated power $\mathrm{kw}$ & 17 & Max power $\mathrm{kw}$ & 25 \\
Rated torque $\mathrm{N} \cdot \mathrm{m}$ & 180 & Max torque $\mathrm{N} \cdot \mathrm{m}$ & 400 \\
\hline
\end{tabular}

(i) velocity: $90 \mathrm{Km} / \mathrm{h}$,

(ii) double lane change test,

(iii) no braking,

(iv) target path: $350 \mathrm{~m}$ straight totally, $3.5 \mathrm{~m}$ bias between $70 \mathrm{~m}$ and $150 \mathrm{~m}$,

(v) road friction coefficient: $\mu=0.85$.

Seen from Figure 6, which is the trajectory of double lane change test, with the comparison among the control one, the no-control one, and the target trace, one can find that the one that used adaptive slid mode control can align with the target path, while a drift occurred at $230 \mathrm{~m}$ and becomes unstable in the no-control one.

Figures 6-9 show the results of handling parameter performances in the double lane change experiment. As shown in Figure 7, the one under control always holds a small side-slip angle; however, the no-control one shows a sharp increase after $33 \mathrm{~s}$. So the same conclusion can be got from

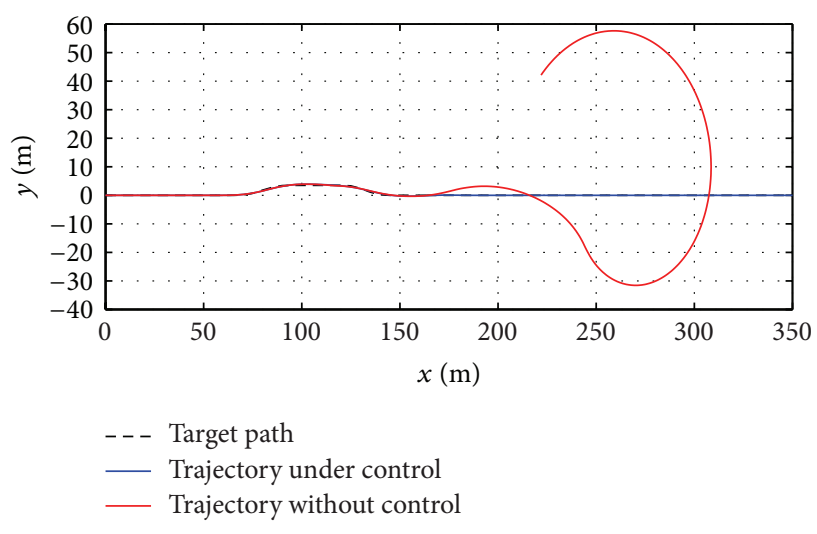

FIGURE 5: Comparison of vehicle trajectory between the one under control and the no-control one.

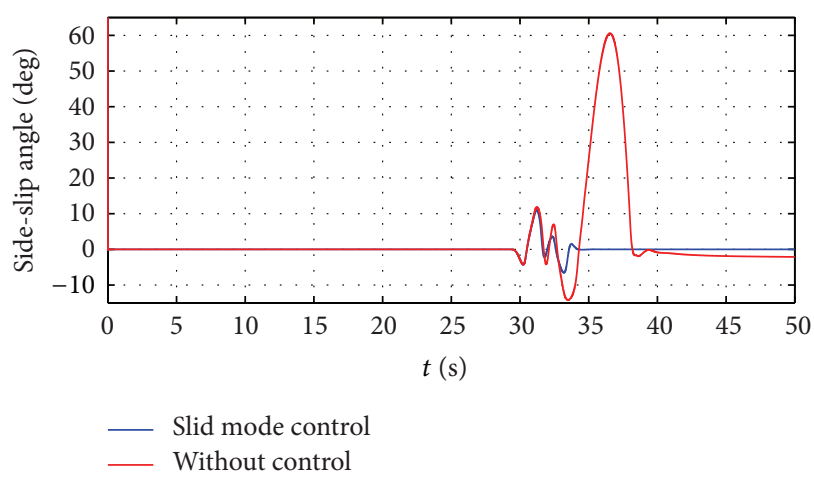

FIGURE 6: Side-slip angle in double lane change test. 


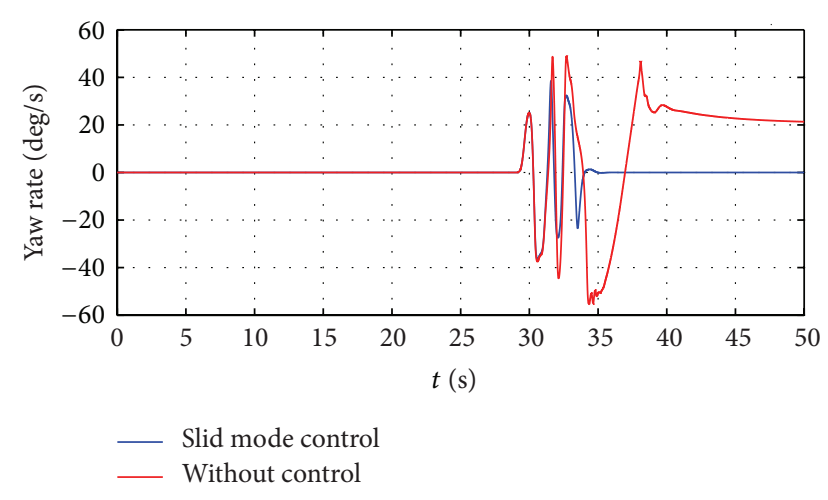

Figure 7: Changing of yaw rate in double lane change test.

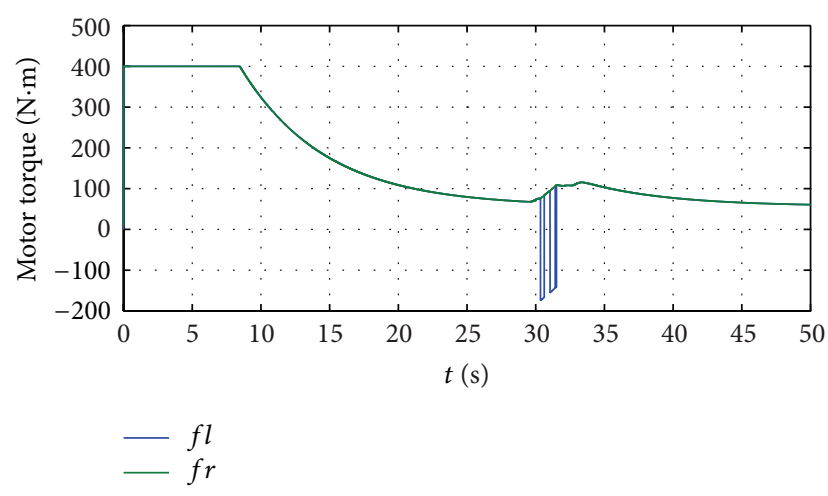

FIGURE 8: Changing of motor torque under slid mode control.

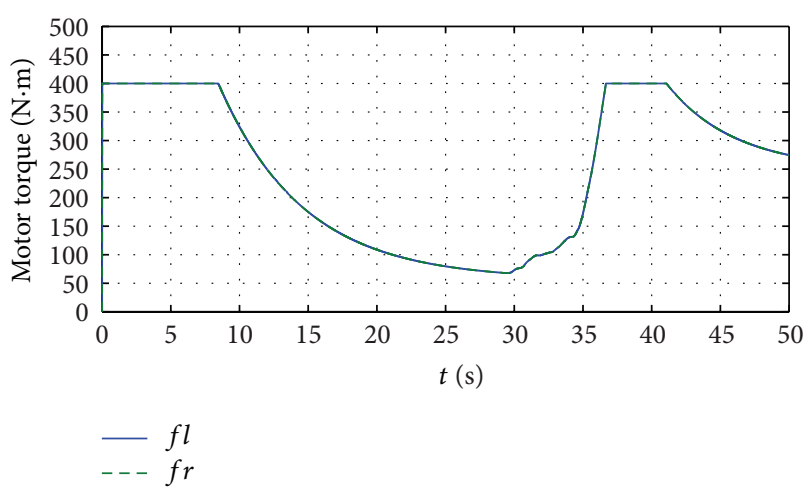

FIGURE 9: Changing of motor torque without control.

Figure 8, which shows the changing of yaw rate. Figures 89 stand for the torque change of the driving motors; we can find that MEV under adaptive slid mode control can tune the output torque reasonably to maintain the vehicle stability, which cannot be implemented in the no-control one.

Figures 10 and 11 vividly show the driving trace of MEV; with the animation one can find that the safety of MEV under control was improved obviously. To conclude, without control, a drift occurred, but with the adaptive slid mode control, a smaller yaw rate and a lower side-slip angle were obtained, and vehicles can align with the trace very well.

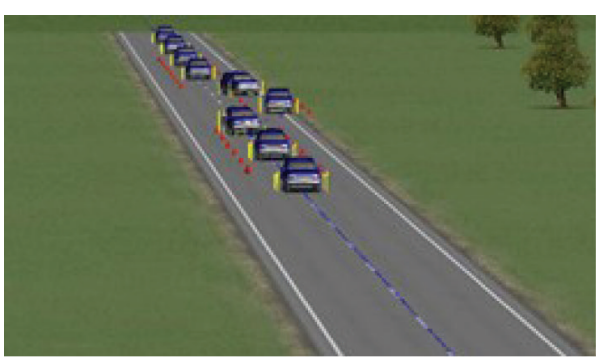

FIgURE 10: Animation of trajectory under slid mode control.

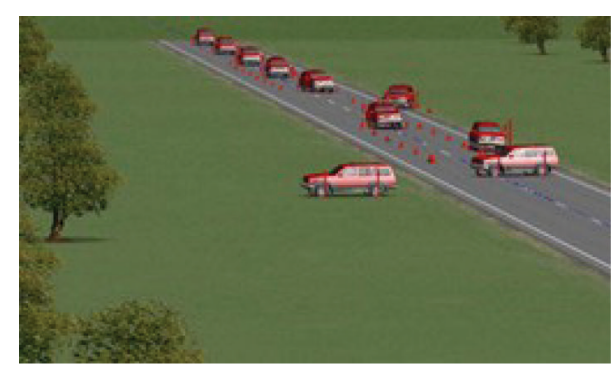

FIGURE 11: Animation of trajectory without control.

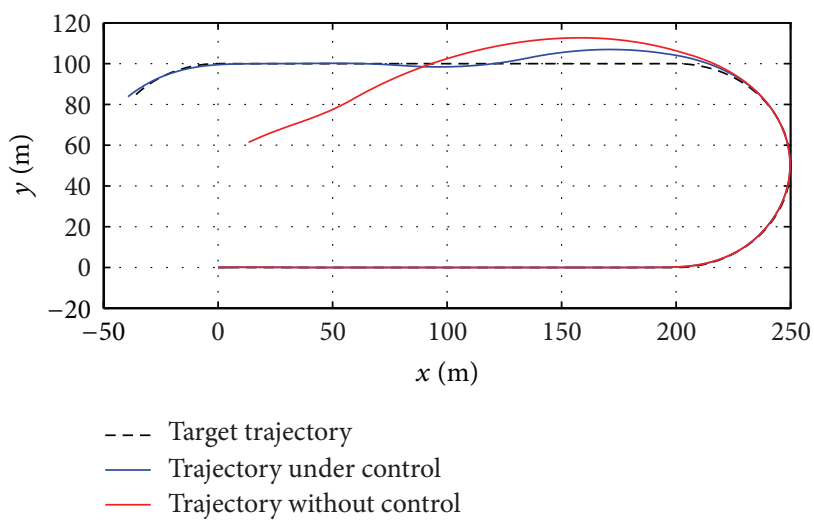

FIGURE 12: Comparison of vehicle trajectory between the one under control and the no-control one.

6.2. U Turn Test. The basic test conditions are listed as follows:

(i) velocity: $50 \mathrm{Km} / \mathrm{h}$,

(ii) "U" turn test,

(iii) braking when needed,

(iv) target path: $200 \mathrm{~m}$ straight, $50 \mathrm{~m}$ radius of half circle, then $200 \mathrm{~m}$ straight,

(v) road friction coefficient: $\mu=0.5$.

Figure 12 shows the vehicle trajectory in $U$ turn test. Vehicle without control was off the target trace, which shows very dangerous working conditions. The one under adaptive slid mode control followed the target path and only gained a small deviation.

The results of parameters were shown in Figures 13-16. Figure 13 shows that the side-slip angle of the no-control one 


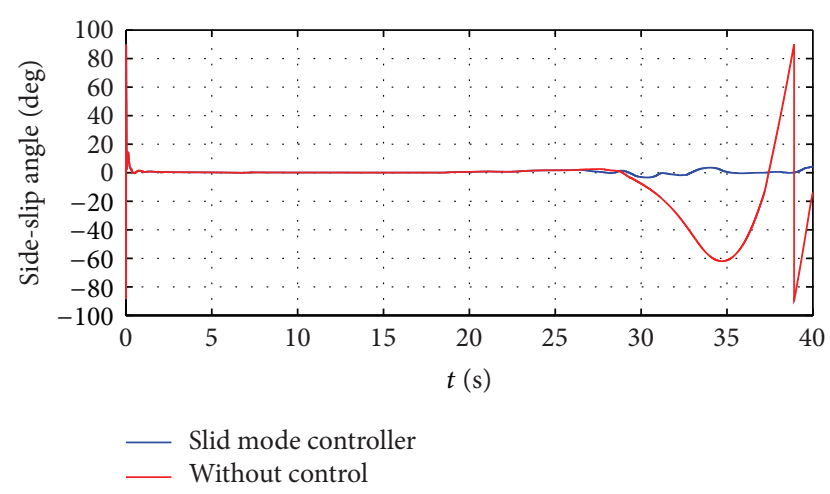

FIGURE 13: Changing of side-slip angle in U test.

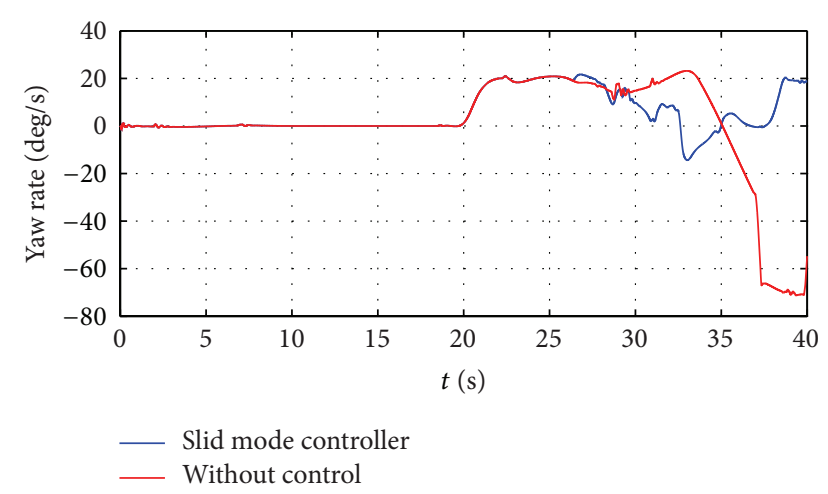

FIGURE 14: Changing of yaw rate in U test.

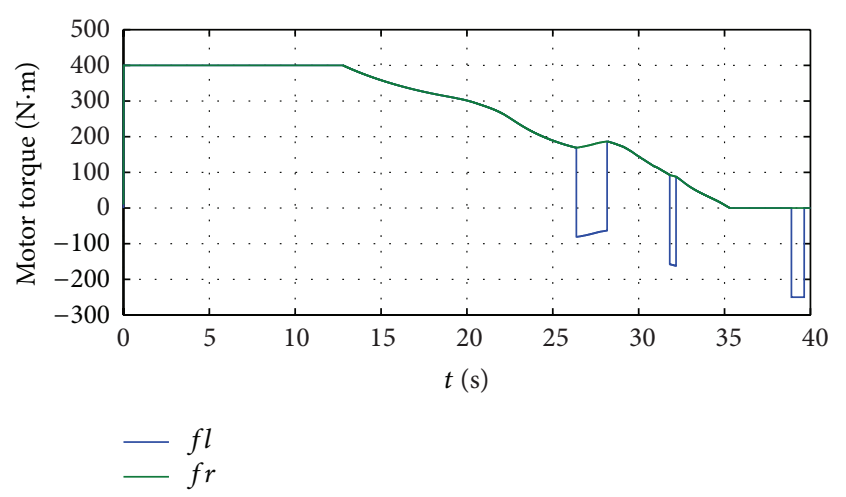

Figure 15: Changing of motor torque under slid mode control.

turns to be a very large value after $30 \mathrm{~s}$. On the contrary, the one under control shows better working conditions. As seen from Figure 14, the yaw rate of the no-control one was soaring and becomes unstable after $32 \mathrm{~s}$, while yaw rate of the one under control is always smaller than the no-control one and also enjoys a good performance. Figures 15 and 16 show the change of motor torques; we can find that the one under control tuned the torque when a bad steering occurred. Finally, the torque value of the one under control turn to be constant, which means the vehicle's speed becomes stable and works well.

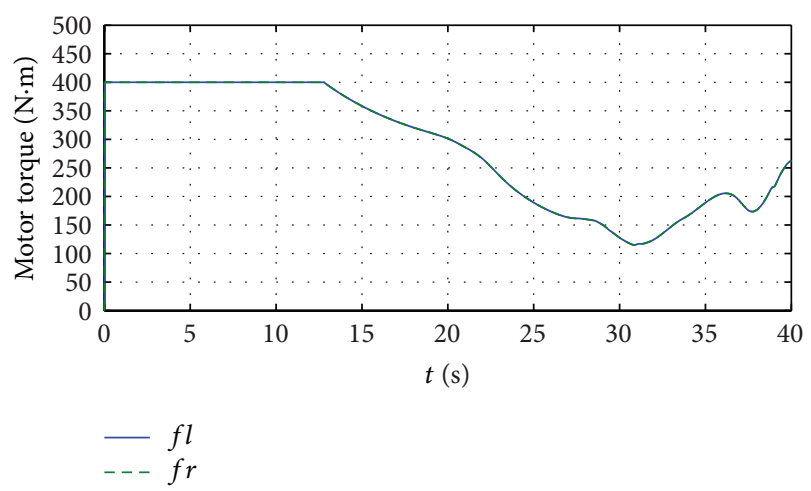

FIGURE 16: Changing of motor torque without control.

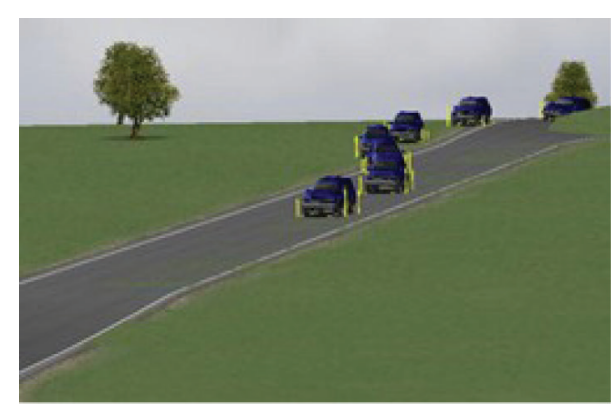

FIGURE 17: Animation of trajectory under slid mode control.

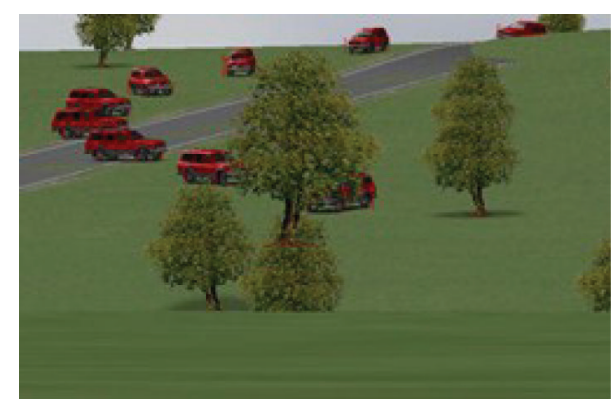

FIGURE 18: Animation of trajectory without control.

Figures 17 and 18 show the trace of MEV lively. With the pictures one can easily know the merits of the adaptive slid mode controller. To sum up, in the $\mathrm{U}$ turn test, the one under control enjoys a smaller yaw rate as well as a lower side-slip angle than the no-control one. The one without control was made to oversteer and become unstable, which may lead to traffic accidents.

Based on the given two comparative vehicle simulation tests, it is undoubted that vehicle driven by in-wheel motors equipped with the adaptive controller can perform well in obstacle avoidance and extreme driving conditions in daily uses. The idea suggested in this study for the in-wheel electric vehicle improved the vehicle handling stability. 


\section{Conclusions}

To sum up, the research is based on MEV platform. An adaptive slid mode control strategy is proposed to evaluate the handling stability of vehicle. A simplified vehicle model is established and deduced, after which a real time estimation method for side-slip angle is also provided based on three basic sensors. A theory for identify road adhesion properties in real-time is researched based on MEV platform for improving control performances. Two kinds of typical handling experiments, double lane change test and $U$ turn test, are also carried out. Based on the vivid comparison of yaw rate, side-slip angle, and trajectory in the tests, one can easily find out that vehicles armed with the adaptive slid mode controller maintain a large enhancement in vehicle stability. In addition, the controller is fit for real-time working circumstances, which will hold practical values in the future. All the work that was studied can illustrate the benefits of motorized electric vehicles (MEV), which will provide a tool in simplifying the cost and the control process; when compared with classical vehicles, the safety of MEV during emergency steering is improved.

\section{Conflict of Interests}

The authors declare that there is no conflict of interests regarding the publication of this paper.

\section{Acknowledgments}

This work is supported by longitudinal project (3R113E482415) and lateral Project (3R110M422415).

\section{References}

[1] P. C. Cho, W. Wylam, and D. Crecelius, "Advanced hybrid electric vehicle propulsion systems with individual wheel brushless traction motors," SAE 2000-01-3110.

[2] S.-K. Chung, H.-S. Kim, C.-G. Kim, and M.-J. Youn, "A new instantaneous torque control of PM synchronous motor for high-performance direct-drive applications," IEEE Transactions on Power Electronics, vol. 13, no. 3, pp. 388-400, 1998.

[3] L.-G. Jin, The Theory and Application of Motorized Wheels for Electric Vehicle, College of Engineering, Jilin University, Changchun, China, 2006.

[4] H. Kataoka, H. Sado, I. Sakai, and Y. Hori, "Optimal slip ratio estimator for traction control system of electric vehicle based on fuzzy inference," Electrical Engineering in Japan, vol. 135, no. 3, pp. 56-63, 2001.

[5] M. Terashima, T. Ashikaga, T. Mizuno, K. Natori, N. Fujiwara, and M. Yada, "Novel motors and controllers for highperformance electric vehicle with four in-wheel motors," IEEE Transactions on Industrial Electronics, vol. 44, no. 1, pp. 28-38, 1997.

[6] G. Freitag, M. Klopzig, K. Schleicher, M. Wilke, and M. Schramm, "High-performance and highly efficient electric wheel hub drive in automotive design," in Proceedings of the IEEE 3rd International Electric Drives Production Conference (EDPC '13), pp. 1-7, Nuremberg, Germany, October 2013.
[7] A. Dadashnialehi, A. Bab-Hadiashar, Z. Cao, and A. Kapoor, "Intelligent sensorless ABS for in-wheel electric vehicles," IEEE Transactions on Industrial Electronics, vol. 61, no. 4, pp. 19571969, 2014.

[8] G. Le Solliec, A. Chasse, J. Van-Frank, and D. Walser, "Dual mode vehicle with in-wheel motor: regenerative braking optimization," Oil and Gas Science and Technology, vol. 68, no. 1, pp. 95-108, 2013.

[9] J. Wang, Q. Wang, L. Jin, and C. Song, "Independent wheel torque control of $4 \mathrm{WD}$ electric vehicle for differential drive assisted steering," Mechatronics, vol. 21, no. 1, pp. 63-76, 2011.

[10] Y. Hori, "Future vehicle driven by electricity and controlresearch on four-wheel-motored 'UOT Electric March II"' IEEE Transactions on Industrial Electronics, vol. 51, no. 5, pp. 954-962, 2004.

[11] P. Khatun, C. M. Bingham, N. Schofield, and P. H. Mellor, "Application of fuzzy control algorithms for electric vehicle antilock braking/traction control systems," IEEE Transactions on Vehicular Technology, vol. 52, no. 5, pp. 1356-1364, 2003.

[12] T. Umeno, E. Ono, K. Asano et al., "Estimation of tire road friction using tire vibration model," SAE paper 2002-01-1183, 2002.

[13] E. Ono, K. Asano, M. Sugai et al., "Estimation of friction force characteristics between tire and road using wheel velocity and application to braking control," DENSO Technical Review, vol. 9, no. 1, pp. 161-168, 2004.

[14] L. Li, K. Shinya, and Y. Hori, "Anti-slip control for EV using dynamic model error based on back-EMF observer," in Proceedings of the Annual Conference of IEEE Industrial Electronics Society (IECON '04), Busan, Korea, 2004.

[15] H. Zhang, X. Zhang, and J. Wang, "Robust gain-scheduling energy-to-peak control of vehicle lateral dynamics stabilisation," Vehicle System Dynamics, vol. 52, no. 3, pp. 309-340, 2014.

[16] Z. Shuai, H. Zhang, J. Wang, J. Li, and M. Ouyang, "Combined AFS and DYC control of four-wheel-independent-drive electric vehicles over CAN Network with time-varying delays," IEEE Transactions on Vehicular Technology, vol. 63, no. 2, pp. 591-602, 2014.

[17] Z. Shuai, H. Zhang, J. Wang, J. Li, and M. Ouyang, "Lateral motion control for four-wheel-independent-drive electric vehicles using optimal torque allocation and dynamic message priority scheduling," Control Engineering Practice, vol. 24, no. 1, pp. 55-66, 2014.

[18] R. Wang, H. Zhang, and J. Wang, "Linear parameter-varyingbased fault-tolerant controller design for a class of over-actuated non-linear systems with applications to electric vehicles," IET Control Theory \& Applications, vol. 8, no. 9, pp. 705-717, 2014.

[19] X. Huang, H. Zhang, G. Zhang, and J. Wang, "Robust weighted gain-scheduling $H_{\infty}$ vehicle lateral motion control with considerations of steering system backlash-type hysteresis," IEEE Transactions on Control Systems Technology, vol. 22, no. 5, pp. 1740-1753, 2014.

[20] Y. Shibahata, K. Shimada, and T. Tomari, "Improvement of vehicle maneuverability by direct yaw moment control," Vehicle System Dynamics, vol. 22, no. 5-6, pp. 465-481, 1993.

[21] A. T. van Zanten, R. Erhardt, and G. Pfaff, "VDC, the vehicle dynamics control system of bosch," SAE 950759, SAE, 1995.

[22] A. T. van Zanten, R. Erhardt, K. Landesfeind, and G. Pfaff, "VDC systems development and perspective," SAE Technical Paper 980235, 1998. 
[23] H. E. Tseng, B. Ashrafi, D. Madau, T. A. Brown, and D. Recker, "The development of vehicle stability control at Ford," IEEE/ASME Transactions on Mechatronics, vol. 4, no. 3, pp. 223234, 1999.

[24] L. Jin, Q. Wang, and H. Zhang, "Experimental study on the differential capability of motorized wheels driving vehicles," China Mechanical Engineering, vol. 18, no. 21, pp. 2632-2636, 2007.

[25] K. Jalali, Stability control of electric vehicles with in-wheel motors [Ph.D. thesis of Philosophy in Mechanical Engineering], University of Waterloo, Waterloo, Canada, 2010.

[26] D. W. Pi, N. Chen, J. X. Wang, and B. J. Zhang, "Design and evaluation of sideslip angle observer for vehicle stability control," International Journal of Automotive Technology, vol. 12, no. 3, pp. 391-399, 2011.

[27] K. Nam, H. Fujimoto, and Y. Hori, "Lateral stability control of in-wheel-motor-driven electric vehicles based on sideslip angle estimation using lateral tire force sensors," IEEE Transactions on Vehicular Technology, vol. 61, no. 5, pp. 1972-1985, 2012.

[28] J. K. Liu and F. C. Sun, "Research and development on theory and algorithms of sliding mode control," Control Theory and Applications, vol. 24, no. 3, pp. 407-418, 2007. 


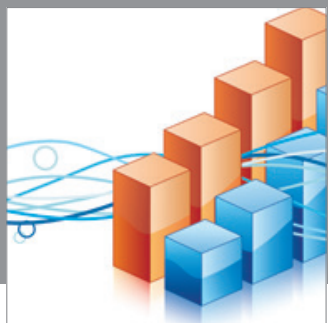

Advances in

Operations Research

mansans

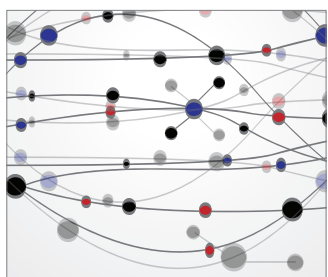

The Scientific World Journal
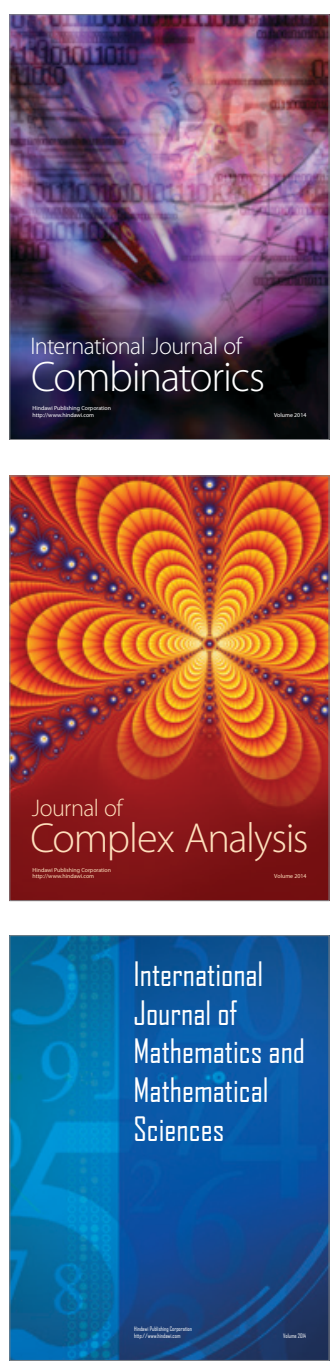
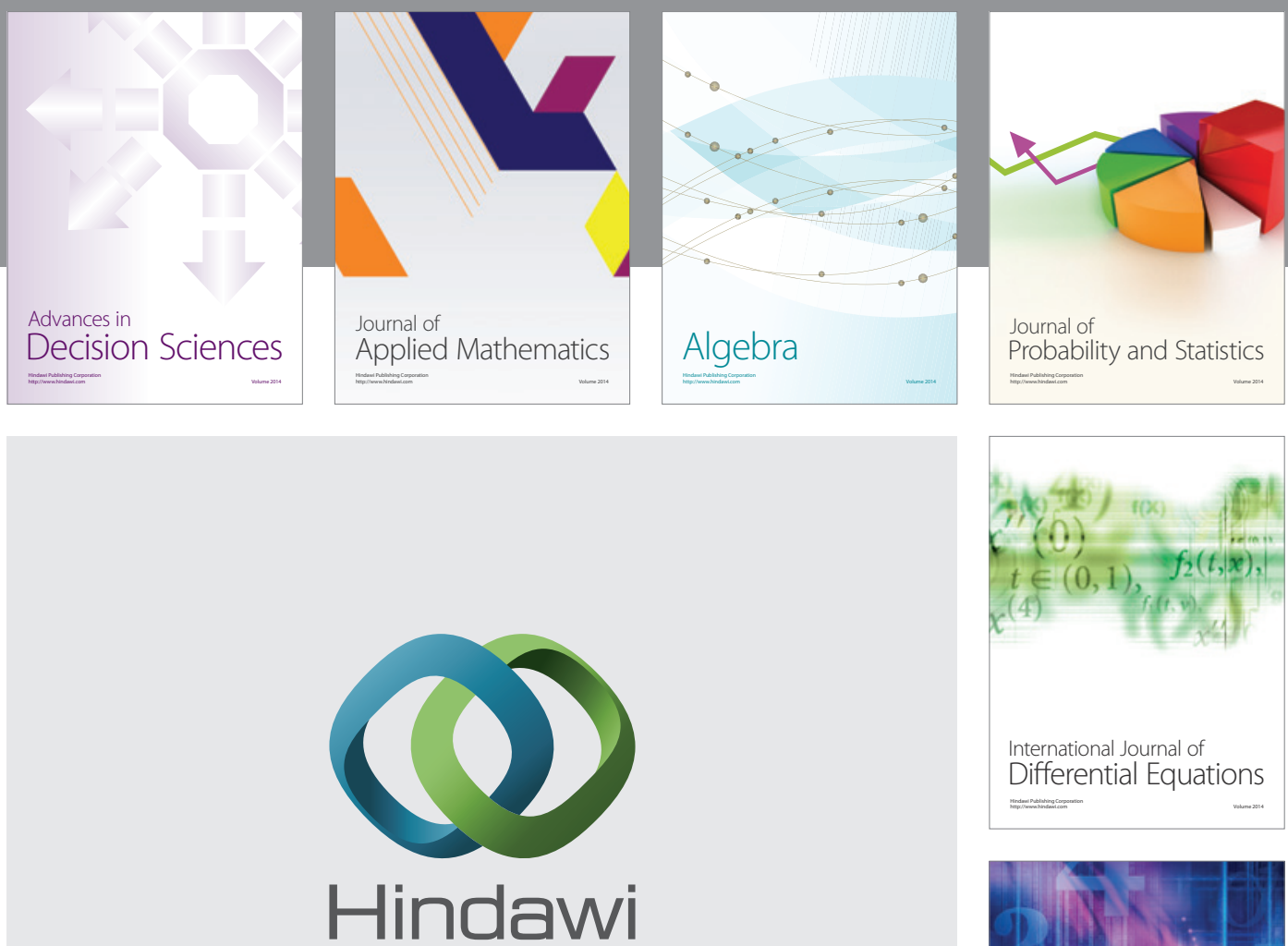

Submit your manuscripts at http://www.hindawi.com
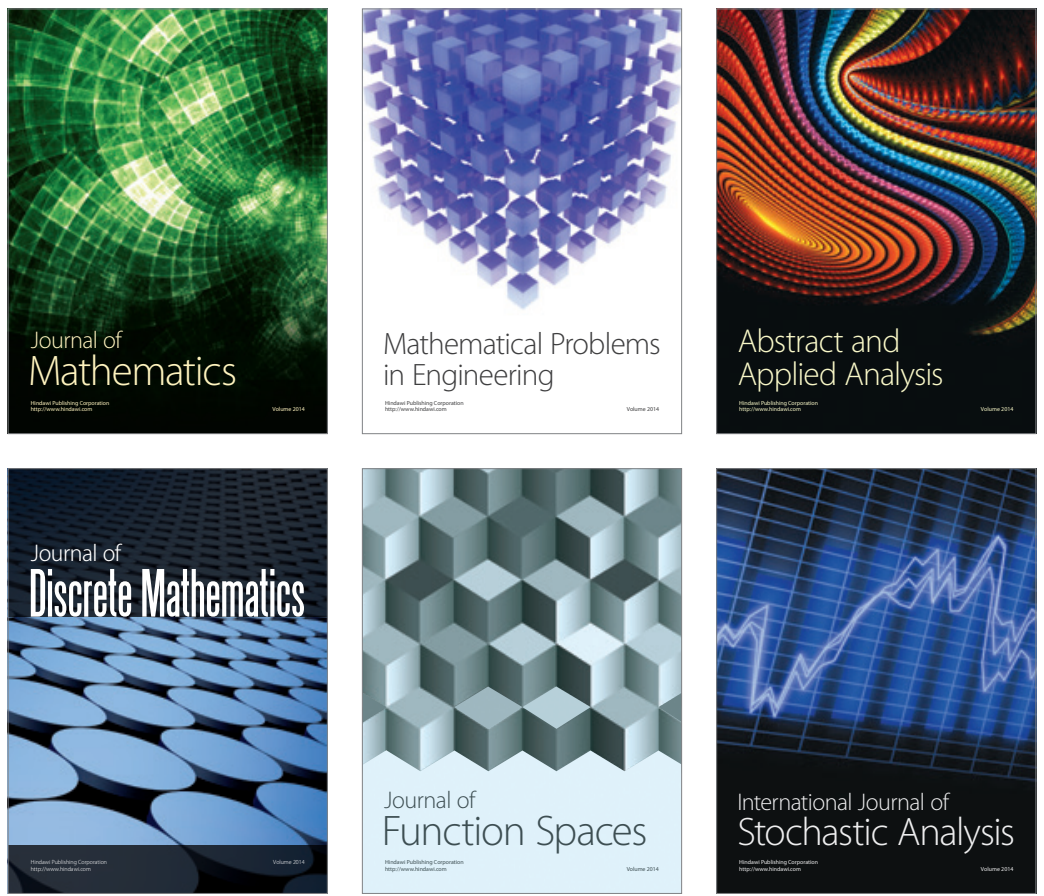

Journal of

Function Spaces

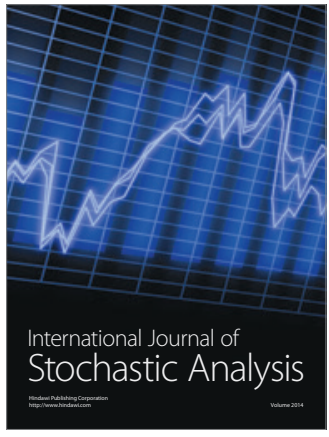

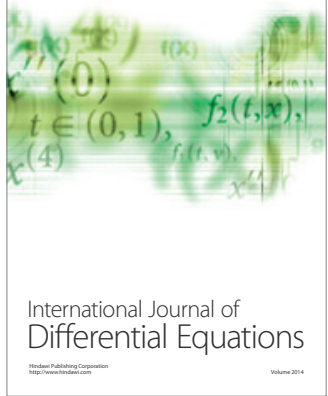
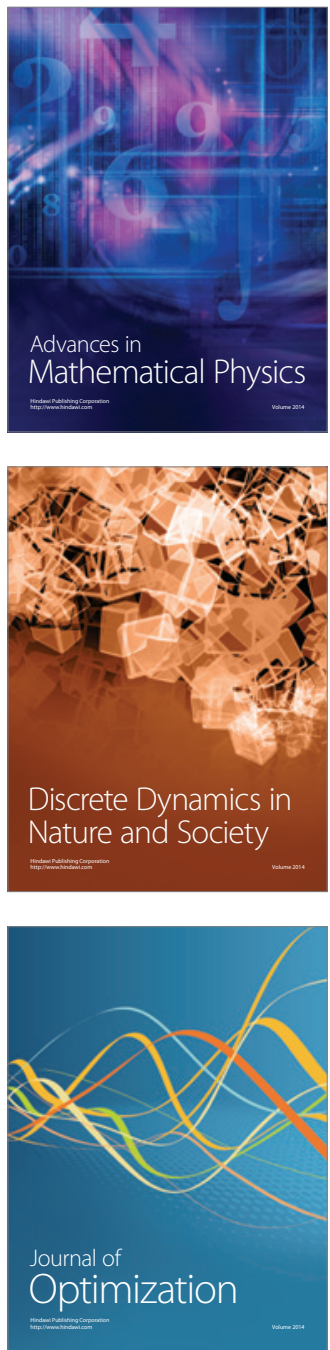